\title{
Como os profissionais da atenção primária percebem e desenvolvem a Educação Popular em Saúde?
}

\author{
Tácia Maria Pereira Flisch(a) \\ Rodrigo Henrique Alves ${ }^{(b)}$ \\ Thiara Amanda Corrêa de Almeida ${ }^{(c)}$ \\ Heloísa de Carvalho Torres ${ }^{(\mathrm{d})}$ \\ Virginia Torres Schall(e) \\ Dener Carlos dos Reis ${ }^{(f)}$
}

Flisch TMP, Alves RH, Almeida TAC, Torres HC, Schall VT, Reis DC. How do primary care professionals perceive and develop Popular Health Education? Interface (Botucatu). 2014;18 Supl 2:1255-1268.

The aim of this study was to analyze the perceptions and experiences of Health Education amongst 166 professionals of Family Health teams in the city of Contagem, State of Minas Gerais, Southeastern Brazil. Participants answered a questionnaire about Health Education practices. When reporting practices aimed at quality of life and human education, they considered issues beyond the informational purpose. All professional categories mentioned greater participation in groups for controlling hypertension and diabetes mellitus. Use of Paulo Freire's pedagogy was reported by $4.7 \%$ of the respondents. Terms indicating political intentionality, such as "social control" and "Popular Health Education", were not mentioned. It was noticed that continuing education processes need to be offered to Family Health professionals and that a national policy for popular Health Education must be implemented.

Keywords: Health Education. Health Promotion. Family Health. Primary Health Care.
O objetivo do estudo foi analisar percepções e experiências em Educação em Saúde de 166 profissionais de equipes de Saúde da Família de Contagem (MG, Brasil), que responderam um questionário sobre práticas de Educação em Saúde. Os participantes consideraram aspectos além da finalidade informativa, ao relatar práticas voltadas à qualidade de vida e à formação humana. Todas as categorias profissionais mencionaram uma maior participação em grupos de controle da hipertensão arterial sistêmica e diabetes mellitus. Utilizam a pedagogia de Paulo Freire 4,7\% dos entrevistados. Termos que demonstram a intencionalidade política, como "controle social" e "Educação Popular em Saúde" não foram mencionados. Foi constatada a necessidade de processos de educação permanente para os profissionais e implementação da política nacional de Educação Popular em Saúde.

Palavras-chave: Educação em Saúde. Promoção da Saúde. Saúde da Família. Atenção Primária à Saúde.

\footnotetext{
"Elaborado com base em Flisch ${ }^{1}$, estudo aprovado pelo Comitê de Ética em Pesquisa da UFMG.

(a,f) Departamento de Enfermagem Materno Infantil e Saúde Pública, Escola de Enfermagem, Universidade Federal de Minas Gerais

(UFMG). Rua Peru, 233,

Eldorado. Contagem, MG, Brasil. 32340-070. taciaflisch@gmail.com; denercarlosreis@yahoo.

(b) Instituto de Ciências Biológicas, UFMG. Belo Horizonte, MG, Brasil. alvesrodrigoh@yahoo.

(c,d) Escola de Enfermagem, UFMG. Belo Horizonte, MG, Brasil.

thiaramanda@hotmail. com; heloisa.ufmg@ gmail.com

(e) Laboratório de Educação em Saúde e Ambiente (LAESA),

Centro de Pesquisa René Rachou, Fundação Oswaldo Cruz. Belo Horizonte, MG, Brasil. vtschall@cpqrr.fiocruz.br
} 


\section{Introdução}

A Educação em Saúde (ES) é uma atividade essencial para a promoção da saúde das populações². Apresenta-se como um campo de teoria e prática interdisciplinar, comprometido com a implementação e avaliação de processos educativos voltados para a promoção da autonomia, da participação e da postura ética, corresponsável e segura dos indivíduos e das comunidades diante de suas questões de saúde e de meio ambiente ${ }^{3,4}$. A Atenção Básica é o nível de atenção à saúde mais profícuo para o desenvolvimento da ES por visar ao desenvolvimento de uma atenção integral que impacte na situação de saúde e autonomia das pessoas e nos determinantes e condicionantes de saúde das coletividades. A Saúde da Família é a estratégia prioritária para a expansão e consolidação da Atenção Básica, que, nas atuais concepções, considera-se equivalente ao termo Atenção Primária à Saúde 5 .

Acredita-se que um caminho possível para potencializar as práticas coletivas de ES, na Atenção Primária à Saúde (APS), seria privilegiar as ações longitudinais. Essas se traduzem em práticas que não se limitam a ações pontuais, pois são conduzidas de forma periódica com o mesmo grupo de participantes. Por essa característica, acredita-se que elas criariam espaços mais permanentes para que os sujeitos possam ressignificar e coproduzir conhecimentos necessários ao alcance do processo de emancipação ou de empoderamento esperados como resultados dessas ações ${ }^{6,7}$.

As ações de ES no Brasil têm, em suas raízes, estratégias tecnicistas, biologicistas e pouco participativas, que privilegiam ações voltadas para um viver higiênico e saudável, direcionadas para as classes populares, vistas como incapazes de iniciativas próprias para a manutenção da saúde individual e coletiva ${ }^{3,8}$.

A mudança desse contexto ocorreu na década de 1970, por meio de uma proposta de medicina comunitária que valorizava os aspectos preventivos da saúde. Nesse período, os profissionais de saúde incorporaram conceitos da pedagogia de Paulo Freire nas ações de ES. Isso resultou em uma relação menos vertical entre os profissionais e a sociedade nessas práticas ${ }^{3}$.

A partir desse cenário, intensificou-se a produção de conhecimentos vinculados à Educação Popular em Saúde (EPS). Hoje, essa área representa um espaço importante de articulação política, de troca de experiências e de formulação de teorias e propostas alternativas para o funcionamento dos serviços de saúde 9,10 .

A EPS favorece a compreensão da realidade no campo da saúde e do meio ambiente e auxilia a população a se organizar para modificá-la. Suas práticas buscam o desenvolvimento de atitudes mais políticas nas comunidades, com a finalidade de ampliar o exercício da autonomia e do controle socia| ${ }^{8,11}$. A política pública de EPS em vigor propõe a participação social como uma diretriz nas ações educativas da APS. Essa perspectiva pressupõe a participação de sujeitos sociais, ativos, criativos, transformadores e tem como missão o apoio ao desenvolvimento de práticas que fortaleçam a constituição desses sujeitos ${ }^{5,12}$. Entretanto, questiona-se se as recomendações dessa política estão sendo implementadas de forma adequada pelos profissionais da APS 3,13 .

Diante desse cenário, o estudo teve como objetivo analisar as percepções e experiências em ES de profissionais da atenção primária à saúde.

\section{Metodologia}

\section{Características do estudo}

Trata-se de um estudo transversal e descritivo com métodos de coleta e análise de dados quantitativos e qualitativos.

Há diversos aspectos que podem influenciar na forma como a ES vem sendo pensada, planejada e operacionalizada no âmbito da APS. A Figura 1 demonstra, de forma esquematizada, que a ES é resultado de diversas dimensões inter-relacionadas, e, dentro de cada uma delas, os seus principais aspectos, que podem influenciar o alcance de seus resultados. Dentre esses aspectos estão os serviços de saúde, os quais envolvem políticas, programas, infraestrutura, materiais e a valoração de práticas 
educativas no cotidiano dos profissionais de saúde. A formação em saúde pode ser decisiva para o desenvolvimento de competências e de incorporação de concepções pedagógicas no campo da saúde. É ressaltada a importância da parceria entre a comunidade e os profissionais que desenvolvem as práticas educativas em saúde.

Educação em Saúde

1 - Serviços de Saúde

a) Organização dos serviços de

saúde

b) Políticas públicas de educação em saúde

c) Programas, ações e linhas de cuidado em Saúde Pública que incluem a educação em saúde

d) Infraestrutura, materiais pedagógicos

e) Valoração das práticas educativas em saúde

f) Percepção dos gestores e dos profissionais de saúde sobre educação em saúde
2 - Produção científica

a) História da educação em saúde - marcos e concepções hegemônicas

b) Elaboração de estruturas conceituais, concepções, processos, materiais e tecnologias

c) Relação teoria $\times$ prática
4 - Comunidade, ambiente e contexto a) Nível de adesão/participação e valoração das práticas de educação em saúde

b) Contexto político, cultural, social e ambiental

c) Modos de viver, de ser saudável, de adoecer: percepções, experiências

d) Nível de conhecimento em saúde

e) Aspectos econômicos e educacionais

Figura 1. Aspectos da prática coletiva de educação em saúde.

\section{Local de estudo}

O estudo foi realizado no município de Contagem, MG, no âmbito da APS nas Unidades Básicas de Saúde, que, no ano de 2011, somavam 88 equipes de saúde da família.

\section{Participantes do estudo}

Participaram do estudo os profissionais que atuam nas ESF, na sua composição mínima: agentes comunitários de saúde, auxiliares e técnicos de enfermagem, enfermeiros e médicos. Para a seleção dos participantes que responderam ao questionário, foi feito um cálculo amostral, visando obter uma amostra estatisticamente representativa da população total de profissionais que atuam nas ESF de Contagem, MG.

No momento do cálculo amostral, a população total de profissionais que atuavam nas 88 ESF era de 663 profissionais distribuídos da seguinte forma: 85 médicos, 85 enfermeiros, 107 profissionais do nível médio de enfermagem e 383 agentes comunitários de saúde. 
O tamanho da amostra que compôs a população de estudo foi calculado considerando uma prevalência de 50\% de prática coletiva de ES na Estratégia Saúde da Família (ESF) e com uma margem de $10 \%$, devido à inexistência de parâmetros na literatura e a grande variabilidade no número de atividades de ES entre as equipes de Saúde da Família do município em estudo, considerou-se também um nível de significância de 5\%. Foi realizado um cálculo amostral, visando obter uma amostra estatisticamente representativa da população total de profissionais $(N=663)$. A amostra estimada foi de 168 profissionais. Adicionaram-se $20 \%$ a esse valor, considerando as possíveis perdas amostrais, resultando em uma amostra de 202 profissionais, divididos em 26 equipes de Saúde da Família, considerando uma média de oito profissionais por equipe. Os critérios de escolha dessas 26 equipes estão explicados a seguir.

Para esse cálculo amostral foi considerada uma amostragem estratificada e por conglomerados, já que o município de Contagem possui sete distritos sanitários com características territoriais e de saúde peculiares em alguns aspectos. Os estratos foram os sete distritos sanitários, com amostra proporcional ao número de equipes por distrito. Foram considerados como conglomerados cada uma das equipes de Saúde da Família, que foram sorteadas, de acordo com o número definido no cálculo amostral, totalizando 26 equipes. Além destas, foi incluída a equipe que participou do teste piloto do estudo, resultando, dessa forma, em 27 equipes de Saúde da Família participantes.

Após a definição do número de equipes por distrito sanitário, foram feitos sorteios aleatórios das equipes que iriam participar. Nos casos de recusa ou impossibilidade da sorteada, os pesquisadores fariam novo sorteio para reposição de perda amostral, o qual não ocorreu, uma vez que todas as equipes sorteadas participaram do estudo.

No momento da entrevista, nem todas as equipes estavam com seu quadro profissional completo. Isso resultou em números diferentes do esperado para algumas categorias profissionais, como médicos e enfermeiros. Dos 202 profissionais esperados para participarem do estudo, 166 responderam ao questionário, incluindo os profissionais da equipe piloto, representando $82,2 \%$ da amostra. Dessa forma, participaram do estudo 166 profissionais das equipes de Saúde da Família, sendo $22(13,3 \%)$ enfermeiros, 17 (10,2\%) médicos, $24(14,5 \%)$ auxiliares e técnicos de enfermagem e 103 (62\%) agentes comunitários de saúde (ACS).

\section{Coleta de dados}

Foi utilizado, como instrumento de coleta de dados, um questionário composto por dez questões estruturadas, em que os participantes escolhiam a resposta mais adequada, dentre as opções padronizadas por meio de um teste piloto. Apesar disso, havia a opção do participante descrever um tipo de resposta diferente das opções predefinidas. O questionário também possuía cinco questões abertas para o relato de aspectos das práticas coletivas de ES desenvolvidas pelos participantes. Essas questões abordaram o conhecimento e a percepção dos participantes sobre as dificuldades e oportunidades, as experiências significativas, os processos de planejamento e operacionalização das ações de ES.

O questionário foi autoaplicável, previamente validado pela realização de um teste piloto, em uma equipe de saúde não sorteada, para verificar o potencial desse instrumento, obter os dados esperados para a pesquisa e realizar as adaptações necessárias previamente à sua aplicação, assim como para o cálculo de tempo de aplicação e a avaliação do entendimento das questões pelos profissionais de saúde. Como não houve mudança das perguntas, os questionários respondidos pela equipe não sorteada integraram o banco de dados do estudo.

\section{Análise dos dados}

As respostas das questões estruturadas do questionário foram processadas no programa estatístico Statistical Package for the Social Sciences (SPSS) (versão 18.0) que permitiu uma análise descritiva, em percentual e número absoluto, das variações das repostas nas opções descritas nessas questões. Utilizou-se o teste do qui-quadrado de Pearson com nível de significância de $p \leq 0,05$, para identificar 
diferenças nos percentuais por tipo de resposta de acordo com a categoria profissional. Trata-se de um estudo descritivo cujo desenho não permite uma análise estatística avançada.

Os dados das questões abertas foram analisados de acordo com os pressupostos da análise de conteúdo de Bardin ${ }^{14}$, método que visa compreender o sentido das informações dadas pelos participantes nos questionários, interpretando as suas significações explícitas e implícitas. Entre as técnicas sugeridas, optou-se por um agrupamento de termos ou expressões-chave com significados semelhantes. A interpretação dos resultados obtidos nesses processos foi fundamentada, principalmente, em referenciais teóricos do campo da EPS apontado na literatura como uma importante área de conhecimento e de prática de atividades educativas coletivas no âmbito da APS 10,12,15,16.

\section{Resultados}

Os resultados demonstraram que, quando questionados sobre o nível de conhecimento em práticas coletivas de ES, todas as categorias profissionais reconheceram a necessidade de aperfeiçoamento.

Somente $28,2 \%$ dos profissionais consideraram ter conhecimento adequado para o desenvolvimento das práticas de ES. Os enfermeiros apresentaram o maior percentual (45\%) de conhecimento satisfatório na área, seguida da categoria médica $(33,3 \%)$, em menores percentuais, os profissionais de nível médio de enfermagem (27,3\%) e os ACSs (23,5\%).

Em concordância com esses resultados, $76,5 \%$ das respostas dos ACSs, $66,7 \%$ dos relatos dos médicos e $72,7 \%$ dos profissionais de enfermagem de nível médio apontaram a necessidade de capacitação para o desenvolvimento dessas práticas (Tabela 1).

Ao serem questionados sobre a fundamentação ou utilização de referencial teórico-pedagógico em ações de ES, os participantes mencionaram, de forma majoritária, que não utilizam referenciais. Eles fazem uso da reprodução de grupos existentes anteriormente na unidade de saúde ou de experiências educativas vivenciadas anteriormente por eles (35\%). Em seguida, foi relatada a utilização de livros de técnicas para trabalhos pedagógicos em grupo (22,3\%), além de práticas simplificadas, sem uma fundamentação teórica $(21,4 \%)$. Não responderam a fundamentação teórica utilizada 13,3\% dos participantes. Apenas 4,7\% responderam que utilizam a pedagogia de Paulo Freire e 2,8\% reconheceram que não há fundamentação teórica nas práticas coletivas (Tabela 1).

Em relação à reprodução de grupos, anteriormente mencionada, seu uso foi relatado, em maior percentual $(42,8 \%)$ pelos profissionais de nível médio da enfermagem, seguidos pelas categorias médica $(40,9 \%)$, enfermeiros $(38,5 \%)$ e ACSs (32\%). A utilização de livros de técnicas para trabalhos pedagógicos em grupo e as práticas sem fundamentação teórica foram relatadas, em maiores percentuais, entre os enfermeiros $(30,8 \%)$ e a categoria médica $(27,3 \%)$, respectivamente. Destaca-se que $19,7 \%$ dos ACSs não responderam o item do questionário sobre a concepção pedagógica utilizada pela equipe de Saúde da Família nas práticas coletivas de ES (Tabela 1).

A categoria médica apresentou o maior percentual $(9,2 \%)$ de menção ao desconhecimento de metodologias pedagógicas que devem ser utilizadas no desenvolvimento de práticas coletivas de ES. Apesar desses percentuais, apenas 1,3\% dos profissionais entrevistados reportaram não apresentarem dificuldade para a realização de práticas de ES no contexto das equipes de Saúde da Família em que atuam (Tabela 1).

Observou-se que, quando solicitados a expressarem a sua percepção sobre a ES no contexto da APS, identificou-se diversidade de palavras/expressões $(\mathrm{N}=307)$ que foram agrupadas em sete categorias temáticas (Tabela 2).

As palavras/expressões que obtiveram maiores percentuais de relato foram as associadas a comportamentos, atitudes e sentimentos (29\%), a exemplo de "ética", "o saber ouvir", "diálogo", "perseverança", "insegurança", "cansativo" e "frustração". Em sua maioria, as palavras/expressões expressam aspectos positivos associados à ES (88\%). A ES como uma prática que promove resultados nas ações de saúde foi mencionada em 9,9\% das respostas, associada a palavras/expressões como "tomada de consciência", "autoestima" e "autonomia" (Tabela 2). 
Tabela 1. Descrição do nível de conhecimento e da fundamentação das práticas coletivas de Educação em Saúde Coletiva, de acordo com a categoria profissional, Contagem, MG (2011).

\begin{tabular}{|c|c|c|c|c|c|}
\hline \multirow[b]{2}{*}{ Categoria } & \multicolumn{5}{|c|}{ Profissionais } \\
\hline & $\begin{array}{l}\text { ACS } \\
\mathrm{N}(\%)\end{array}$ & $\begin{array}{c}\text { Nível médio enfermagem } \\
\qquad \mathrm{N}(\%)\end{array}$ & $\begin{array}{l}\text { Enfermeiro } \\
\qquad \mathrm{N}(\%)\end{array}$ & $\begin{array}{l}\text { Médico } \\
\text { N (\%) }\end{array}$ & $\begin{array}{l}\text { Total } \\
\mathrm{N}(\%)\end{array}$ \\
\hline \multicolumn{6}{|l|}{ Nível de conhecimento*,\# } \\
\hline Precisa melhorar & $65(76,5)$ & $16(72,7)$ & $11(55,0)$ & $10(66,7)$ & $102(71,8)$ \\
\hline Adequado & $20(23,5)$ & $6(27,3)$ & $9(45,0)$ & $5(33,3)$ & $40(28,2)$ \\
\hline \multicolumn{6}{|l|}{ Referencial teórico\#,"** } \\
\hline Reprodução de grupos & $39(32,0)$ & $12(42,8)$ & $15(38,5)$ & $9(40,9)$ & $75(35,5)$ \\
\hline Livros de técnicas & $23(18,8)$ & $6(21,4)$ & $12(30,8)$ & $6(27,3)$ & $47(22,3)$ \\
\hline Intuitiva & $26(21,3)$ & $7(25,0)$ & $6(15,4)$ & $6(27,3)$ & $45(21,4)$ \\
\hline Não sabe responder & $24(19,7)$ & $1(3,6)$ & $2(5,1)$ & $1(4,5)$ & $28(13,3)$ \\
\hline Concepções de Paulo Freire & $5(4,1)$ & $1(3,6)$ & $4(10,2)$ & - & $10(4,7)$ \\
\hline Não há & $5(4,1)$ & $1(3,6)$ & - & - & $6(2,8)$ \\
\hline
\end{tabular}

"Excluídos 24 questionários sem essa informação; \# Valores de p >0,05 (não significativo - teste qui-quadrado de Pearson); "“Fundamentação da prática coletiva de educação em saúde.

Tabela 2. Descrição das palavras/expressões associadas à Educação em Saúde de acordo com a categoria temática, Contagem, MG (2011).

\begin{tabular}{|c|c|c|}
\hline Categorias temáticas & $\mathrm{N}(\%)$ & Exemplos de termos/expressões \\
\hline $\begin{array}{l}\text { Educação como expressão } \\
\text { de atitudes }\end{array}$ & $89(29,0)$ & $\begin{array}{l}\text { Ética - coparticipação - saber ouvir - diálogo - respeito - perseverança - falta } \\
\text { de comprometimento - insegurança - cansativo - frustração - insatisfação. }\end{array}$ \\
\hline $\begin{array}{l}\text { Educação para o alcance } \\
\text { de resultados em saúde }\end{array}$ & $61(19,9)$ & $\begin{array}{l}\text { Tomada de consciência - responsabilização - fortalecimento de vínculos } \\
\text { - aprendizagem sobre doença - diminuição do número de consultas - } \\
\text { autoestima - autonomia. }\end{array}$ \\
\hline Educação por temas de saúde & $43(14,0)$ & $\begin{array}{l}\text { Planejamento familiar - pré-natal - vida sexual para adolescentes - Doença } \\
\text { Sexualmente Transmissível (DST) - Diabetes Mellitus (DM) - Hipertensão } \\
\text { Arterial Sistêmica (HAS). }\end{array}$ \\
\hline $\begin{array}{l}\text { Educação para o ensino, cuidado } \\
\text { e formação humana }\end{array}$ & $36(11,7)$ & $\begin{array}{l}\text { Aprendizado multidisciplinar - educação permanente - crescimento coletivo - } \\
\text { troca de saberes - educação da comunidade. }\end{array}$ \\
\hline $\begin{array}{l}\text { Educação e sua variação de } \\
\text { formato, público e local }\end{array}$ & $33(10,7)$ & $\begin{array}{l}\text { Ação em grupo e individual - grupos educativos - sala de espera - cartilhas - } \\
\text { família - atividade na comunidade- visitas. }\end{array}$ \\
\hline $\begin{array}{l}\text { Educação para planejamento } \\
\text { em saúde }\end{array}$ & $30(9,8)$ & $\begin{array}{l}\text { Motivação entre usuário e equipe - acesso - melhor aproveitamento do } \\
\text { tempo - reconhecimento profissional - falta de capacitação profissional - } \\
\text { falta de apoio da gestão. }\end{array}$ \\
\hline $\begin{array}{l}\text { Educação associada à qualidade } \\
\text { de vida }\end{array}$ & $15(4,9)$ & $\begin{array}{l}\text { Exercício e atividade física - lazer - passeios - cuidados - mudança de hábito } \\
\text { - bem-estar físico e mental - convivência. }\end{array}$ \\
\hline Total & $307(100)$ & \\
\hline
\end{tabular}

Expressões como a "hipertensão arterial", "diabetes mellitus", "saúde da mulher e da criança", além de "limpeza do domicílio" e "lavagem das mãos" foram destaque na categoria de educação por temas de saúde (14\%). Já na categoria que englobou a educação em sua variabilidade de formato, local e público, foram citadas as palavras/expressões como "ações em escolas", "ações em UBS", "grupos temáticos", "ações individuais" e "família". A ES como processo de ensino, cuidado e formação humana foi relacionada a termos antagônicos, como por exemplo, "informar/transmitir 
conhecimentos" contrapondo "troca de conhecimentos/experiências". A categoria qualidade de vida descreve termos que sugerem o bem-estar das pessoas, como convivência, lazer e passeio (Tabela 2).

Cabe mencionar que os resultados da categoria ES como recurso para o planejamento incluiu aspectos positivos e negativos. Os primeiros podem ser exemplificados pelas palavras/expressões como "favorecer o melhor aproveitamento do tempo" e "promover o reconhecimento profissional". Exemplificam os aspectos negativos as "deficiências na área de capacitação profissional" e a "falta de recursos" (Tabela 2). Ressalta-se que as palavras/expressões menos referidas foram associadas às categorias de ES para planejamento em saúde $(9,8 \%)$ e educação associada à qualidade de vida (4,9\%) (Tabela 2$)$.

O tipo de prática educativa apresenta uma predominância de grupos educativos relacionados ao programa de controle da hipertensão arterial sistêmica (HAS) e diabetes mellitus (DM), independentemente da categoria. Destaca-se o maior percentual de participação dos ACSs e dos profissionais de nível médio em enfermagem em grupos de puericultura, com 55\% e 62,5\%, respectivamente. Grupos voltados para a melhoria da qualidade de vida, como grupos de caminhada, convivência, idosos, produção de artesanatos e saúde do homem, raramente, ou nunca, em algumas ESF, tem a participação de médicos. Embora menos frequentes, há diversos outros grupos citados pelos participantes, como saúde da mulher, adolescentes, planejamento familiar, alimentação saudável, saúde mental (Tabela 3).

\section{Discussão}

A ES, apesar de toda a sua especificidade, é, em essência, educação. Como prática social humana, a educação é um processo histórico, contínuo, que emerge da dialética entre homem, mundo, história e circunstâncias. A sua finalidade na área da saúde é promover patamares mais elevados de autonomia, de corresponsabilização por meio da reflexão crítica, para os sujeitos identificarem e intervirem sobre as questões de saúde e meio ambiente, o que lhe outorga o caráter de atividade complexa ${ }^{8,17}$.

Apesar de a politicidade ser um dos pilares da prática de ES na APS, observou-se que não há uma visão unívoca da $\mathrm{ES}^{12}$. Esse resultado pode sugerir que essa percepção diferenciada seja produto das diferenças na formação profissional nessa área e na valoração que cada profissional atribui à prática educativa. Ainda permanece ativa uma prática educativa pouco inovadora e, muitas vezes, afastada do conhecimento científico produzido nessa área e da política nacional nesse campo de atuação.

A literatura científica aponta para uma possível naturalização das práticas educativas, o que significa dizer que, em muitas situações, não se busca trazer novas concepções pedagógicas e variações das práticas de ES comumente realizadas ${ }^{3,18}$. Tal naturalização pode criar obstáculos para a composição de uma política de educação permanente na área que inclua novas abordagens e metodologias as quais favoreçam a efetivação da política nacional de EPS, no nível local3,18,19.

Em consonância com essa discussão, a maioria dos participantes reconheceu a necessidade de ampliação dos seus conhecimentos sobre EPS. Diante desse resultado e das diferenças nas percepções sobre ES entre as categorias profissionais investigadas, é possível apontar alguns desafios para as práticas de ES no âmbito da APS. Entre eles, está a necessidade de qualificação e de formação dos profissionais da ESF para atuar em ações que visem à obtenção de melhorias na qualidade de vida dos indivíduos e populações, considerando que a saúde é também produto do contexto social e ambiental. A ES exige, portanto, ações voltadas para o desenvolvimento de atitudes politizadas desses profissionais e também da população alvo das práticas educativas ${ }^{19}$.

A necessidade de ampliar os conhecimentos em ES, apontada pelos participantes do estudo, vai ao encontro do cenário nacional, que busca qualificar as ações de ES por meio da implantação da Política Nacional de Educação Popular que tem como uma de suas perspectivas a construção da autonomia e emancipação dos grupos populacionais historicamente excluídos de seu modo de entender a vida e em seus saberes ${ }^{12}$.

Esses resultados podem indicar também a importância da ampliação de disciplinas curriculares na graduação que favoreçam uma formação adequada dos diversos profissionais de saúde. Essa ampliação visa a criar competências e habilidades para as práticas coletivas de $\mathrm{ES}^{20}$. 
Tabela 3. Tipo de práticas coletivas de educação em saúde coletiva, de acordo com a categoria profissional, Contagem, MG (2011).

\begin{tabular}{|c|c|c|c|c|}
\hline \multirow[b]{2}{*}{$\begin{array}{l}\text { Prática } \\
\text { tipo/frequência }\end{array}$} & \multicolumn{4}{|c|}{ Profissionais } \\
\hline & $\begin{array}{l}\text { ACS } \\
\mathrm{N}(\%)\end{array}$ & $\begin{array}{l}\text { Nível médio enfermagem } \\
\qquad \mathrm{N}(\%)\end{array}$ & $\begin{array}{l}\text { Enfermeiro } \\
\quad \mathrm{N}(\%)\end{array}$ & $\begin{array}{l}\text { Médico } \\
\mathrm{N}(\%)\end{array}$ \\
\hline \multicolumn{5}{|l|}{ HAS } \\
\hline Frequente & $63(64.2)$ & $17(70,8)$ & $10(45,5)$ & $9(53,0)$ \\
\hline Raro/nunca & $35(35,8)$ & $7(29,2)$ & $12(54,5)$ & $8(47,0)$ \\
\hline \multicolumn{5}{|l|}{ DM } \\
\hline Frequente & $61(62,2)$ & $16(66,7)$ & $12(54,5)$ & $8(47,0)$ \\
\hline Raro/nunca & $37(37,8)$ & $8(33,3)$ & $10(45,5)$ & $9(53,0)$ \\
\hline \multicolumn{5}{|l|}{ Puericultura } \\
\hline Frequente & $54(55,0)$ & $15(62,5)$ & $6(27,3)$ & $5(29,4)$ \\
\hline Raro/nunca & $44(45,0)$ & $9(37,5)$ & $16(72,7)$ & $12(70,6)$ \\
\hline \multicolumn{5}{|l|}{ Gestante } \\
\hline Frequente & $53(54,0)$ & $8(33,3)$ & $10(45,5)$ & $6(35,3)$ \\
\hline Raro/nunca & $45(46,0)$ & $16(66,7)$ & $12(54,5)$ & $11(64,7)$ \\
\hline \multicolumn{5}{|l|}{ Controle peso } \\
\hline Frequente & $36(36,7)$ & $10(41,6)$ & $11(50,0)$ & $4(23,5)$ \\
\hline Raro/nunca & $62(63,3)$ & $14(58,4)$ & $11(50,0)$ & $13(76,5)$ \\
\hline \multicolumn{5}{|l|}{ Saúde mental } \\
\hline Frequente & $30(30,6)$ & $6(25)$ & $7(31,8)$ & $5(29,4)$ \\
\hline Raro/nunca & $68(69,4)$ & $18(75)$ & $15(68,2)$ & $12(70,6)$ \\
\hline \multicolumn{5}{|l|}{ Qualidade vida } \\
\hline Frequente & $15(15,4)$ & $3(12,5)$ & $4(18,2)$ & - \\
\hline Raro/nunca & $83(84,6)$ & $21(87,5)$ & $18(81,8)$ & $17(100)$ \\
\hline \multicolumn{5}{|l|}{ Convivência } \\
\hline Frequente & $11(11,3)$ & $2(8,3)$ & $5(22,7)$ & $1(5,0)$ \\
\hline Raro/nunca & $87(88,7)$ & $22(91,7)$ & $17(77,3)$ & $16(95,0)$ \\
\hline \multicolumn{5}{|l|}{ Idosos } \\
\hline Frequente & $10(10,2)$ & $3(12,5)$ & $3(13,6)$ & - \\
\hline Raro/nunca & $88(89,8)$ & $21(87,5)$ & $19(86,4)$ & $17(100)$ \\
\hline \multicolumn{5}{|l|}{ Atividade física } \\
\hline Frequente & $6(6,1)$ & $3(12,5)$ & $4(18,2)$ & - \\
\hline Raro/nunca & $92(93,9)$ & $21(100)$ & $18(81,8)$ & $17(100)$ \\
\hline \multicolumn{5}{|l|}{ Artesanato } \\
\hline Frequente & $10(10,2)$ & $4(16,6)$ & $3(13.6)$ & - \\
\hline Raro/nunca & $88(88,8)$ & $20(83,3)$ & $19(86,4)$ & $17(100)$ \\
\hline \multicolumn{5}{|l|}{ Passeio } \\
\hline Frequente & $5(26,7)$ & $1(4,2)$ & - & - \\
\hline Raro/nunca & $93(73,3)$ & $23(95,8)$ & $22(100)$ & $17(100)$ \\
\hline \multicolumn{5}{|l|}{ Asma } \\
\hline Frequente & $5(26,7)$ & $1(4,2)$ & - & - \\
\hline Raro/nunca & $93(73,3)$ & $23(95,8)$ & $22(100)$ & $17(100)$ \\
\hline \multicolumn{5}{|l|}{ Saúde homem } \\
\hline Frequente & $3(3,1)$ & $3(12,5)$ & - & - \\
\hline Raro/nunca & $95(96,9)$ & $21(100)$ & $22(100)$ & $17(100)$ \\
\hline \multicolumn{5}{|l|}{ Outros } \\
\hline Frequente & $12(12,2)$ & $6(25)$ & $9(40,1)$ & $2(11,8)$ \\
\hline Raro/nunca & $86(87,8)$ & $18(75)$ & $13(59,9)$ & $15(88,2)$ \\
\hline
\end{tabular}

$\mathrm{N}=161$. HAS (hipertensão arterial sistêmica). DM (diabetes mellitus). Outros (leishmaniose, planejamento familiar, sala de espera, resultados de prevenção de câncer de colo uterino, obesidade, relaxamento, adolescentes, educação sexual, crianças de baixo peso, saúde da mulher, culinária e dança).

Nesse sentido, o estudo apontou que o conhecimento nessa área ocorre de forma desigual nos cursos da área da saúde. Como exemplo, os enfermeiros relataram ter um nível de conhecimento maior de ES que os outros profissionais. Isso pode ser explicado pela sua maior percepção de que a ação educativa é inerente à prática do cuidado. Além disso, esse profissional geralmente atua no 
planejamento e desenvolvimento dessas práticas educativas em saúde, o que pode estimular a busca pelo conhecimento nesse domínio ${ }^{21}$.

Em relação às percepções dos entrevistados sobre a ES, no que se refere a sua variação de formato, público e local, foram mencionadas expressões que remetem aos projetos e programas de base comunitária. De fato, esses programas representam uma importante abordagem no contexto da APS, ao combinar a produção de conhecimentos e a ação para a mudança social, visando a eliminar as iniquidades em saúde. Isso vai ao encontro a um dos pressupostos da política de EPS, que é o de promover o ativismo das pessoas e dos grupos sociais e incentivar seu envolvimento em programas sociais que visam à ampliação da autonomia e ao empoderamento dos participantes ${ }^{22-25}$.

Outra percepção encontrada no estudo se refere à constatação de que os profissionais entrevistados mencionaram que utilizam a reprodução de grupos nas ações educativas das ESF. Essas ações, em geral, são conduzidas sem fundamentação pedagógica adequada ${ }^{26}$. Essa percepção pode levar a um afastamento do reconhecimento de que tais práticas educativas precisam ser planejadas, com base em seu contexto de atuação e fundamentadas em referenciais pedagógicos da área da educação. Para a viabilização dessas práticas, são necessários investimentos em educação permanente dos profissionais, materiais pedagógicos, adequação de infraestrutura, representando, portanto, um desafio para a gestão dos serviços na APS.

As percepções relacionadas à educação, como uma estratégia para o planejamento em saúde, indicam a necessidade de uma melhor gestão das ações que devem ser desenvolvidas pela equipe de saúde, além de atividades que motivem a participação de usuários e equipe nessas práticas. Nesse âmbito, o desafio é motivar e valorar a participação da comunidade e dos profissionais de saúde ${ }^{16}$.

Os fundamentos da pedagogia de Paulo Freire ${ }^{27}$ apresentaram um baixo percentual de menção nas práticas de EPS no cenário pesquisado, apesar do seu reconhecimento internacional como uma abordagem importante para a promoção da saúde e para movimentos sociais, além de ser um fundamento importante da política nacional da APS ${ }^{12}$.

Há um reconhecimento de que essa pedagogia favorece o exercício do controle social e de projetos comunitários voltados para o aperfeiçoamento dos serviços de saúde e de melhorias das condições de vida dos indivíduos.

Além disso, mesmo quando a pedagogia de Paulo Freire foi mencionada pelos profissionais de saúde como fonte para o planejamento e desenvolvimento de suas ações em EPS, sua utilização foi controversa. Isso, provavelmente, em decorrência do seu emprego simplificado, considerando tão somente o diálogo, com a troca de informações entre profissionais e usuários e de uma investigação da realidade sem a apropriação da estrutura conceitual e analítica e sem a intencionalidade política próprias dessa abordagem ${ }^{15}$.

Considerando-se o emprego simplificado dessa pedagogia, pode ocorrer a exclusão, no processo educativo, dos aspectos relacionados à realidade do indivíduo ou comunidade. Isso pode comprometer a necessidade de investigar e refletir sobre a realidade vivenciada, não oportunizando aos indivíduos a expressão dos principais determinantes de uma dada situação na qual estão envolvidos. Como consequência, pode haver o afastamento da fase de aproximação do saber do mundo da vida com o saber técnico-científico, de forma crítica e reflexiva.

Para a mudança da realidade observada neste estudo, sugere-se a promoção de eventos, em âmbito local, regional e nacional, com ênfase no compartilhamento de experiências de EPS. Podem ser priorizadas as experiências que considerem uma educação fundamentada no diálogo, na promoção da reflexão crítica, da interação dos diferentes saberes e dos modos de interpretar a realidade de saúde de uma comunidade, conforme preconiza a pedagogia de Paulo Freire ${ }^{12}$.

Além da pedagogia freiriana, o campo da educação possui um vasto elenco de autores que podem contribuir, mediante suas concepções pedagógicas, para a EPS, mas que não foram citados pelos profissionais de saúde. Entre esses, podem ser citados Dewey ${ }^{28}$, Freinet ${ }^{29}$, Vygotsky ${ }^{30}$ os quais apontam que o ensino-aprendizado deve ser fundamentado na experiência e na construção social do conhecimento. Da mesma forma, os princípios pedagógicos defendidos por Deleuze ${ }^{31}$ demonstram que educar é suscitar acontecimentos que levem as pessoas a atribuir significados e sentidos às situações e eventos objetivos/subjetivos do seu cotidiano. 
Além desses autores, as concepções de Hannah Arendt ${ }^{32}$, quando interpretadas no contexto dos processos pedagógicos, partem da premissa de que há uma nítida distinção entre os processos de pensar e conhecer, destacando a importância da reflexão da informação e da ação, a partir da realidade objetiva e subjetiva dos sujeitos envolvidos no ato educacional. Ressalta-se, por fim, o pensamento de Pedro Demo ${ }^{11}$ sobre a politicidade como um compromisso para a educação na atualidade. Todos esses autores podem contribuir para a renovação das práticas de EPS.

Conforme ressaltado anteriormente, a pouca utilização dessas concepções pedagógicas aqui referidas pode ser resultado de lacunas na formação e da não priorização do aprofundamento dessas pedagogias na educação permanente em saúde ${ }^{33}$. Estudos demonstram que a adoção de concepções teórico-filosóficas é fator que norteia a prática de EPS ${ }^{34}$. Possibilitar aos profissionais de saúde o conhecimento de processos pedagógicos pode evitar a continuidade de uma prática unívoca da educação como um processo de "adquirir" e "receber" a informação. Essa prática unívoca pode fazer com que sejam relegadas outras dimensões presentes no ato de educar-aprender, como afetividade, subjetividade, intersubjetividade, na relação do sujeito consigo mesmo, com o outro, com o conhecimento e com a realidade ${ }^{35}$.

O argumento central da EPS é a aproximação e a troca de conhecimento entre equipe e comunidade, propiciando o compartilhamento de ideias e concepções de vida diferentes. O resultado esperado é o alcance de práticas educativas efetivas. Esse desafio está em sintonia com a discussão de Valla ${ }^{36}$ ao apontar que essa ausência de troca efetiva de experiências no ato educacional em saúde gera erros de interpretação da realidade de saúde do indivíduo e da comunidade, já que cada um desses atores sociais pode possuir lógicas e práticas distintas nas formas de conduzir as questões relacionadas à saúde.

As categorias temáticas formadas a partir da descrição de palavras/expressões associados à ES nos permite perceber o vasto alcance de interpretação que essas práticas contêm. Constatou-se que os participantes do estudo mencionaram aspectos que envolvem a subjetividade e a relação interpessoal (atitudes), ao gerenciamento do cuidado (resultado, formato, público, local, planejamento em saúde), à ampliação do ato de cuidar (ensino, cuidado e formação humana), à diminuição de riscos (temas) e à promoção da saúde e do cuidado ambiental (qualidade de vida). Esse resultado foi diferente do esperado. Acreditava-se que as práticas populares em ES estariam centralizadas em atos pedagógicos de prevenção de doenças e agravos à saúde.

Ao contrário, os profissionais demonstraram possuir um potencial para incorporar dimensões que ultrapassam essas práticas informativas. Entre elas, houve relatos de uma ES que favorece a expressão de sentimentos e que contribui para uma formação cidadã. Em outras palavras, as equipes de Saúde da Família trilham um processo que transita entre as práticas mais informativas/tradicionais e práticas mais dialógicas e participativas.

Observa-se que essa transição pode indicar que os participantes do estudo já incorporam novos elementos na prática de EPS. Isso é coerente com os pressupostos e eixos estratégicos da EPS. Dentre esses, podem ser citados a problematização, construção compartilhada do conhecimento, emancipação, compromisso com a construção do projeto democrático e popular, participação, controle social e gestão participativa, formação, comunicação e produção de conhecimento, cuidado em saúde, intersetorialidade e diálogos multiculturais ${ }^{12,37}$.

Essa evidência também indica que a definição de ES é reconstruída de forma contínua na sua prática, pois indica "novos elementos" que devem compor o seu escopo conceitual, uma vez que as palavras e expressões identificadas como associados à ES ultrapassam a definição encontrada no campo teórico 3,12,38,39.

Para se trabalhar com a subjetividade das pessoas, com aspectos culturais e históricos das comunidades, são necessários conhecimentos elaborados, várias leituras e boas experiências educativas. Isso pode evitar a banalização de aspectos fundamentais para a emancipação política dos indivíduos ${ }^{8,16}$.

Os resultados indicam que, apesar da transição da forma como percebem a EPS, os profissionais de saúde parecem não valorizar de modo mais central a promoção de ações que permitam a comunidade desenvolver um pensamento crítico e reflexivo da realidade, levando a uma maior politicidade, em consonância com a política de EPS. Muitas vezes, tais profissionais utilizam palavras e expressões da EPS, tais como "diálogo" e "troca de experiência", porém isso não significa o compromisso com a politicidade 11,27,40. 
Defende-se que temas relacionados à cidadania, autonomia, ética, controle social, deveriam ser transversais às temáticas dos diversos programas inseridos na APS ${ }^{41}$. Além disso, essas temáticas devem considerar as necessidades de saúde das pessoas da comunidade ${ }^{30,42}$.

A associação das práticas educativas, desenvolvidas pelas equipes ESF investigadas, permitiu observar que, em sua maioria, seguem as diretrizes que compõem os principais programas de Atenção Primária à Saúde, a exemplo dos grupos de HAS e DM.

Apesar da realização de práticas que atendem algumas das políticas de Atenção a Saúde em âmbito nacional, observou-se que as equipes de ESF buscam inovar as ações educativas, introduzindo no processo pedagógico atividades que possibilitam a troca de experiências, de informações e permitem o desenvolvimento de habilidades de interação social como dança, grupos de convivência, de produção de artesanatos e passeios programados.

Toda essa análise nos conduz a uma reflexão sobre a importância da relação entre os aspectos que são determinantes para o desenvolvimento das práticas de EPS. Como exemplo, podemos citar a relação entre profissionais de saúde, comunidade, estrutura organizacional dos serviços de saúde, e a efetiva implementação, em âmbito local, da política de EPS $37,43,44$.

\section{Considerações finais}

Este estudo permitiu realizar uma análise das percepções dos profissionais de saúde acerca da EPS. Foi demonstrado que houve diferenças nas percepções e no desenvolvimento de práticas de ES em relação às diferentes categorias profissionais que compuseram as equipes de Saúde da Família, no cenário investigado. Apesar dessas diferenças, houve predominância, entre todas as categorias, de relatos sobre a realização de práticas educativas voltadas para o controle da HAS e DM.

Observou-se também que as práticas de ES são, em geral, desenvolvidas de forma reprodutiva, ou seja, representam apenas uma continuidade de ações que já são conduzidas rotineiramente na APS. Além disso, essas práticas não eram fundamentadas por concepções pedagógicas e nem se apoiavam nas diretrizes da política nacional de ES. Somado a isso, foi observado um baixo percentual de referência à pedagogia de Paulo Freire.

As percepções dos profissionais da ESF sobre a Educação Popular em Saúde na APS indicaram uma fase transicional, ao apontarem elementos que compõem uma prática ainda centrada em difundir informações sobre saúde e doença, vinculadas aos principais programas da APS, mas, ao mencionar em paralelo que realizam práticas coletivas voltadas para potencializar a autonomia e o bem-estar das pessoas, diante dos problemas de saúde individual e coletiva. Porém, esse potencial de mudança deve ser mais bem investigado. O estudo demonstra ainda que há necessidade de maior envolvimento da comunidade nas ações educativas das equipes de Saúde da Família no cenário estudado.

Em geral, os desafios apontados pelo estudo aglutinam-se em torno da necessidade de formação e educação permanente dos profissionais da APS para atuarem em práticas de ES condizentes com as diretrizes da política de EPS. Cabe mencionar que as escolas técnicas e as universidades da área da saúde precisam estar atentas à formação de competências e habilidades dos profissionais para atuarem em práticas educativas.

Enfim, o estudo indica que a percepção sobre a ES se mostra abrangente e com aspectos coerentes com a política nacional de EPS. Ao contrário das práticas de ES mencionadas pelos participantes, que ainda permanecem, em sua maioria, enraizadas em ações educativas tradicionais da APS.

A valoração dessa prática também é desafio para a gestão dos serviços de saúde, no âmbito da APS, no município investigado. Espera-se que haja maiores investimentos na implementação da política de EPS entre as equipes de Saúde da Família, para favorecer o desenvolvimento de competências e habilidades relacionadas à investigação, planejamento e atuação nas práticas coletivas em ES.

A partir dos achados do estudo, recomenda-se a criação de uma política municipal de ES, tendo como referência a EPS, dada a sua importância para os programas e ações da APS.

Reconhece-se que o estudo, apesar de desenvolvido em um município de grande porte do estado de Minas Gerais e retratar alguns aspectos de uma percepção regional, traz elementos que fazem eco à Política Nacional de Educação Popular em Saúde, que são comuns às equipes de Saúde da Família no Brasil. 
Dessa forma, acredita-se que seus resultados podem favorecer debates e integrar referências a processos de educação permanente, ao provocar reflexões acerca das percepções dos profissionais de saúde, com enfoque na necessidade de valorização da EPS dentro do elenco de atividades desenvolvidas pelas equipes de Saúde da Família. Sugere-se, por fim, que outros estudos aprofundem o debate da EPS, no âmbito da APS, com abrangência regional ou nacional, investigando os processos de educação e formação profissional, bem como as barreiras que impedem a efetiva implementação da EPS em nível local.

\section{Colaboradores}

Tácia Maria Pereira Flisch e Dener Carlos dos Reis coletaram e analisaram os dados e participaram da elaboração do texto. Heloísa de Carvalho Torres, Virginia Torres Schall e Rodrigo Henrique Alves participaram da análise dos resultados e da construção do manuscrito. Thiara Amanda Corrêa de Almeida responsabilizou-se pelo processamento dos dados.

\section{Referências}

1. Flisch TMP. Práticas coletivas de educação em saúde na Atenção Primária à Saúde em Contagem, MG [dissertação]. Belo Horizonte (MG): Universidade Federal de Minas Gerais; 2013.

2. Torres HC, Monteiro MRP. Educação em saúde sobre doenças crônicas nãotransmissíveis no programa saúde da família em Belo Horizonte/MG. Rev Min Enferm. 2009; 10(4):397-401.

3. Reis DC. Educação em saúde: aspectos históricos e conceituais. In: Gazzinelli MF, Reis DC, Marques RC. Educação em saúde: teoria, método e imaginação. Belo Horizonte: UFMG; 2006. p. 19-94.

4. Schall VT, Struchiner M. Educação em saúde: novas perspectivas. Cad Saude Publica. 1999; 15 Supl 2:S4-6.

5. Portaria n. 2488, de 21 de outubro de 2011. Aprova a Política Nacional de Atenção Básica, no âmbito do Sistema Único de Saúde. Brasília (DF); 2011.

6. Torres HC, Amaral MA, Amorim MM, Cyrino AP, Bodstein R. Capacitação de profissionais de atenção primária à saúde para educação em diabetes mellitus. Acta Paul Enferm. 2010; 23(6):751-6.

7. Ayres JRCM. Sujeito, intersubjetividade e práticas de saúde. Cienc Saude Colet. 2001; 6(1):63-72.

8. Gomes LB, Merhy EE. Compreendendo a educação popular em saúde: um estudo na literatura brasileira. Cad Saude Publica. 2011; 27(1):7-18.

9. Stotz EN. A Educação popular nos movimentos sociais da saúde: uma análise de experiências nas décadas de 1970 e 1980. Trab Educ Saude. 2005; 3(1):9-20.

10. Stotz EN, David HMSL. Wong Un JA. Educação popular em saúde: trajetória, expressões e desafios de um movimento social. Rev APS. 2005; 8(1):49-60.

11. Demo P. Politicidade: razão humana. Campinas: Papirus; 2002.

12. Ministério da Saúde. Secretaria de Gestão Estratégica e Participativa. Departamento de Apoio à Gestão Participativa. Caderno de educação popular e saúde. Brasília (DF): MS; 2007. 
13. Gazzinelli MF, Gazzinelli A, Reis DC, Penna CMM. Educação em saúde: conhecimentos, representações sociais e experiências da doença. Cad Saude Publica. 2005; 21(1):200-6.

14. Bardin L. Análise de conteúdo. São Paulo: Edições 70; 2011.

15. Wiggins N. Popular education for health promotion and community empowerment: a review of the literature. Health Promot Int. 2011; 27(3):356-71.

16. Pekelman R. Caminhos para uma ação educativa emancipadora: a prática educativa no cotidiano dos serviços de atenção primária em saúde. Rev APS. 2008; 11(3):295-302.

17. Alves VS. Um modelo de educação em saúde para o programa saúde da família: pela integralidade da atenção e reorientação do modelo assistencial. Interface (Botucatu). 2005; 9(16):39-52.

18. Gastaldo D. É a educação em saúde "saudável"? Repensando a educação em saúde através do conceito de bio-poder. Educ Real. 1997; 22(1):147-68.

19. Ceccim RB, Ferla AC. Educação e saúde: ensino e cidadania como travessia de fronteiras. Trab Educ Saude. 2009; 6(3):443-56.

20. Machado MFAS, Monteiro EMLM, Queiroz DT, Vieira NFC, Barroso, MGTI. Integralidade, formação de saúde, educação em saúde e as propostas do SUS: uma revisão conceitual. Cienc Saude Colet. 2007; 12(2):335-42.

21. Campos GWS, organizador. A clínica do sujeito: por uma clínica reformulada e ampliada. In: Saúde Paidéia. São Paulo: Hucitec; 2003. p. 51-68.

22. Parker EA, Chung LK, Israel BA, Reyes A, Wilkins D. Community organizing network for environmental health: using a community health development approach to increase community capacity around reduction of environmental triggers. J Prim Prev. 2010; 31(12):41-58.

23. Ndekha A, Hansen EH, Molgaard P, Woelk G, Furu P. Community participation as an interactive learning process: experiences from a schistosomiasis control project in Zimbabwe. Acta Trop. 2003; 85(3):325-38.

24. Israel BA, Schulz AJ, Parker EA, Becker AB. Review of community-based research: assessing partnership approaches to improve public health. Annu Rev Public Health. 1998; 19:173-202.

25. Dias JCP. Problemas e possibilidades de participação comunitária no controle das grandes endemias no Brasil. Cad Saude Publica. 1998; 14 Supl 2:19-37.

26. Albuquerque $P C$, Stotz EN. A educação popular na atenção básica à saúde no município: em busca da integralidade. Interface (Botucatu). 2004; 8(15):259-74.

27. Freire P. Pedagogia da autonomia: saberes necessários à prática educativa. São Paulo: Paz e Terra; 2002.

28. Teitelbaum K, Apple M. John Dewey. Curriculo sem Front. 2001; 1(2):194-201.

29. Freinet C. Pedagogia do bom senso. 7a ed. São Paulo: Martins Fontes; 2004.

30. Sirgado AP. O social e o cultural na obra de Vigotski. Educ Soc. 2000; 21(71):45-78.

31. Gallo S. Deleuze e a educação. 2a ed. Belo Horizonte: Autêntica; 2008.

32. Almeida VS. A distinção entre conhecer e pensar em Hannah Arendt e sua relevância para a educação. Educ Pesqui. 2010; 36(32):853-65.

33. Villa E. Educação em saúde: a prática educativa no cotidiano do trabalho do profissional. In: Gazzinelli MF, Reis DC, Marques RC, organizadores. Educação em saúde: teoria, método e imaginação. Belo Horizonte: UFMG; 2006. p. 43-51. 
34. Vasconcelos M, Grillo MJ, Soares SM. Práticas pedagógicas em atenção básica à saúde: tecnologias para abordagem ao indivíduo, família e comunidade. Unidade didática I: organização do processo de trabalho na atenção básica à saúde. Belo Horizonte: UFMG; 2009.

35. Demo P. Educação e conhecimento: relação necessária, insuficiente e controversa. Petrópolis: Vozes; 2000.

36. Valla VV. Educação popular, saúde comunitária e apoio social numa conjuntura de globalização. Cad Saude Publica. 1999; 15 Supl 2:7-14.

37. Vasconcelos EM. Educação popular: de uma prática alternativa a uma estratégia de gestão participativa das políticas de saúde. Physis. 2004; 14(1):67-83.

38. Ministério da Saúde. Bases para a educação em saúde nos serviços. Documento preliminar a ser submetido a processo de discussão e aperfeiçoamento na oficina nacional de educação em saúde nos serviços do SUS. Brasília (DF): MS; 2008.

39. Acioli S, David HMSL. A educação em saúde e a enfermagem em saúde coletiva. In: Santos I, David HMSL, Silva D, organizadores. Enfermagem e campos de prática em saúde coletiva: realidade, questões e soluções. São Paulo: Atheneu; 2008. p. 3-8.

40. Gadotti M. Educação comunitária e economia popular. In: Gadotti M, Gutiérrez F, organizadores. Questões de nossa época. 3a ed. São Paulo: Cortez; 2001.

41. Ministério da Saúde. Educação em saúde. Bol Inst Saude. 2004; (34):13-4.

42. Moreira MA. A teoria da aprendizagem significativa de Ausubel. In: Moreira MA, organizador. Teorias da aprendizagem. São Paulo: EPU; 1999. cap. 10, p. 151-65.

43. Roecker S, Nunes EFPA, Marcon SS. Trabalho educativo do enfermeiro na estratégia saúde da família.Texto Contexto Enferm. 2013; 22(1):157-65.

44. Lima KA, Costa FN. A. Educação em saúde e pesquisa qualitativa: relações possíveis. Alim Nutr. 2006; 16(1):33-8.

Flisch TMP, Alves RH, Almeida TAC, Torres HC, Schall VT, Reis DC. ¿Cómo los profesionales de la atención primaria perciben y desarrollan la Educación Popular en Salud? Interface (Botucatu). Interface (Botucatu). 2014;18 Supl 2:1255-1268.

El objetivo del estudio fue analizar percepciones y experiencias en Educación en Salud de 166 profesionales de equipos de Salud de la Familia de Contagem (Estado de Minas Gerais) que respondieron un cuestionario sobre prácticas de Educación en Salud. Los participantes consideraron aspectos más allá de la finalidad informativa, al relatar prácticas enfocadas en la calidad de vida y en la formación humana. Todas las categorías profesionales mencionaron una mayor participación en grupos de control de la hipertensión arterial sistémica y diabetes mellitus. El 4,7\% de los entrevistados utilizan la pedagogía de Paulo Freire. No se mencionaron términos que demuestran la intencionalidad política, tales como "control social" y "Educación Popular en Salud". Se constató la necesidad de procesos de educación permanente para los profesionales e implementación de la política nacional de Educación Popular en Salud.

Palabras-clave: Educación en Salud. Promoción de la Salud. Salud de la Familia. Atención Primaria de la Salud. 